\title{
ESTIMATES OF THE LEAST PRIME FACTOR OF A BINOMIAL COEFFICIENT
}

\author{
P. ERDÖS, C. B. LACAMPAGNE, AND J. L. SELFRIDGE \\ Dedicated to the memory of D. H. Lehmer
}

\begin{abstract}
We estimate the least prime factor $p$ of the binomial coefficient $\left(\begin{array}{l}N \\ k\end{array}\right)$ for $k \geq 2$. The conjecture that $p \leq \max (N / k, 29)$ is supported by considerable numerical evidence. Call a binomial coefficient good if $p>k$. For $1 \leq i \leq k$ write $N-k+i=a_{i} b_{i}$, where $b_{i}$ contains just those prime factors $>\bar{k}$, and define the deficiency of a good binomial coefficient as the number of $i$ for which $b_{i}=1$. Let $g(k)$ be the least integer $N>k+1$ such that $\left(\begin{array}{l}N \\ k\end{array}\right)$ is good. The bound $g(k)>c k^{2} / \ln k$ is proved. We conjecture that our list of 17 binomial coefficients with deficiency $>1$ is complete, and it seems that the number with deficiency 1 is finite. All $\left(\begin{array}{l}N \\ k\end{array}\right)$ with positive deficiency and $k \leq 101$ are listed.
\end{abstract}

\section{GOOD BINOMIAL COEFFICIENTS}

Consider a sequence of $k \geq 2$ positive integers $\{n+i\}=n+1, \ldots, n+k$, with $n+i=a_{i} b_{i}$, where $p \mid a_{i}$ implies $p \leq k$, and $p \mid b_{i}$ implies $p>k$ for any prime $p$; i.e., the $a_{i}$ have the prime factors up to $k$, and the $b_{i}$ have all the larger prime factors.

Since the binomial coefficient $\left(\begin{array}{c}n+k \\ k\end{array}\right)$ is an integer, $k ! \mid \prod a_{i}$. We are concerned with the least prime factor of any number in the sequence, except for those composing $k$ ! , that is, the least prime factor of $\left(\begin{array}{c}n+k \\ k\end{array}\right)$.

Definition 1A. A sequence of consecutive integers $a_{1} b_{1}, \ldots, a_{k} b_{k}$, where $p \mid a_{i}$ implies $p \leq k$, and $p \mid b_{i}$ implies $p>k$, is said to be good if $\prod a_{i}=k$ ! .

We denote the least prime factor of $m$ by $p(m)$. We frequently find it convenient to write $N=n+k$.

Definition 1B. The binomial coefficient $\left(\begin{array}{l}N \\ k\end{array}\right)$ is said to be good if $p\left(\left(\begin{array}{l}N \\ k\end{array}\right)\right)>k$.

Note that Definition 1B is equivalent to stating that the $k$-sequence $\{N-$ $k+i\}$ is good or that $\prod a_{i}=k$ ! or that $\operatorname{gcd}\left(\left(\begin{array}{l}N \\ k\end{array}\right), k !\right)=1$. Further, when $k$ is fixed and $\left(\begin{array}{l}N \\ k\end{array}\right)$ is good, we say that $N$ is good with respect to $k$.

Ecklund, Erdös, and Selfridge [1] studied the function $g(k)$, the least integer $N>k+1$ such that $\left.p\left(\begin{array}{l}N \\ k\end{array}\right)\right)>k$, so $g(k)$ is the least $N$. which is good with respect to $k$. They showed that $g(k)>2 k$ for $k>4$ and established weak upper and lower bounds on $g(k)$. They determined $g(k)$

Received by the editor July 27, 1992 and, in revised form, December 11, 1992..

1991 Mathematics Subject Classification. Primary 11B65, 11N37. 
TABLE 1. Relative minima and maxima of $g(k), k \leq 149$

\begin{tabular}{rrrrrrr}
\multicolumn{2}{c}{ Relative minima } & \multicolumn{2}{c}{ Maxima } & \multicolumn{2}{c}{ Maxima } \\
$k$ & $d>0$ & $g(k)$ & $k$ & $g(k)$ & $k$ & $g(k)$ \\
2 & & 6 & 2 & 6 & 61 & 2237874623 \\
4 & 2 & 7 & 3 & $7^{*}$ & 71 & 3184709471 \\
5 & 1 & 23 & 5 & $23^{*}$ & 72 & 4179979724 \\
8 & 2 & 44 & 6 & $62^{*}$ & 73 & 15780276223 \\
10 & 3 & 46 & 7 & $143^{*}$ & 74 & 19942847999 \\
11 & 4 & 47 & 9 & 159 & 75 & 48899668971 \\
12 & 2 & 174 & 12 & $174^{* *}$ & 83 & 797012560343 \\
14 & 2 & 239 & 13 & 2239 & 89 & 3524996442239 \\
16 & 3 & 241 & 17 & 5849 & 103 & 5092910127863 \\
28 & 9 & 284 & 20 & 43196 & 104 & 6003175578749 \\
33 & 3 & 6459 & 24 & 193049 & 107 & 6260627365739 \\
35 & & 37619 & 29 & 240479 & 108 & 9746385386989 \\
40 & & 85741 & 31 & 341087 & 109 & 73245091349869 \\
42 & 2 & 96622 & 32 & $371942^{*}$ & 110 & 94794806842238 \\
52 & & 366847 & 38 & 487343 & 111 & 222261611307119 \\
58 & & 4703099 & 39 & 767919 & 113 & 517968108138869 \\
99 & 5675499 & 41 & $3017321^{*}$ & 114 & 598199028602614 \\
100 & 3935600486 & 43 & 24041599 & 115 & 12714356616655615 \\
102 & 175209712494 & 44 & 45043199 & 139 & 25972027636644319 \\
106 & 488898352367 & 47 & 232906799 & 141 & 63331523816662671 \\
135 & 3157756005623 & & & & \\
136 & 4138898693368 & & & & \\
148 & 11808400809148 & $d=d(g(k), k)$ & $* d=1$ & $* * d=2$
\end{tabular}

for $2 \leq k \leq 40$ and $k=42,46,52$, and showed by direct search that $g(k)>2.5 \times 10^{6}$ for all other $k \leq 100$. After discussion with us, Scheidler and Williams [5] found all values of $g(k)$ for $k \leq 140$, using the new open architecture sieve at the University of Manitoba. The list of these values appears in [5], and the sieving continues. In Table 1 we present $g(k)$ where $g(k) \leq g(t)$ for $k<t<150$ and where $g(k) \geq g(t)$ for $t<k$. No doubt, $g(k)$ increases faster than polynomially and surely $g(k)<(1+c)^{\pi(k)}$, but we have no proof. When $k$ is large, it is clear to every right-thinking person that $\left(\begin{array}{l}N \\ k\end{array}\right)$ has a prime factor in $(k / 2, k)$ for every $N<\exp (c k / \ln k)$. It seems that $g(k)$ increases very irregularly, and no doubt,

$$
\lim \sup g(k+1) / g(k)=\infty, \quad \liminf g(k+1) / g(k)=0 .
$$

Note that $g(29) / g(28)>846$, and $g(99) / g(98)<1 / 1872$.

Up to $k=148, g(k+1)-g(k)=0$ or 1 only at $k=3,10,18$ and 36 .

It was shown in [1] that there is an absolute constant $c>0$ for which $g(k)>k^{1+c}$. Since $c$ is small, the following is an improved lower bound.

Theorem 1. There holds $g(k)>c_{1} k^{2} / \ln k$, for some absolute constant $c_{1}>0$. Proof. We first show that if $k^{7 / 4} \leq N<c_{1} k^{2} / \ln k$, where $k>k_{0}\left(c_{1}\right)$, then $\left(\begin{array}{l}N \\ k\end{array}\right)$ has a prime factor $p$ satisfying

$$
k / 2<p<k / 2+k^{3 / 4} \text {. }
$$

Begin by noting that Ingham [3] proved that the number of primes $p$ satisfying 
(1) is greater than $k^{3 / 4} /\left(c_{2} \ln k\right)$ for some absolute constant $c_{2}$. By an averaging argument, there are two primes $p_{1}$ and $p_{2}$ satisfying

$$
k / 2<p_{1}<p_{2}<p_{1}+c_{2} \ln k<k / 2+k^{3 / 4}
$$

and $p_{1}<k / 2+k^{3 / 4}-2$. Now we show that for $k^{7 / 4} \leq N<c_{1} k^{2} / \ln k$, either $p_{1}$ or $p_{2}$ divides $\left(\begin{array}{c}n+k \\ k\end{array}\right)$. Let $t p_{1}$ be the largest multiple of $p_{1}$ which is less than or equal to $n$, so that $n<(t+1) p_{1}$. It follows immediately from (2) that if $t p_{1} \leq n-2 k^{3 / 4}+4$, then $(t+2) p_{1} \leq n+k$. Thus $p_{1} \mid\left(\begin{array}{c}n+k \\ k\end{array}\right)$. Therefore, we can assume

$$
t p_{1}>n-2 k^{3 / 4}+4 \text {. }
$$

We wish to show that $t p_{2}>n$ and $(t+1) p_{2} \leq n+k$, which imply that $p_{2} \mid\left(\begin{array}{c}n+k \\ k\end{array}\right)$.

Observe that $p_{1} \leq k$ and $k\left(k^{3 / 4}-1\right)=k^{7 / 4}-k \leq n<(t+1) p_{1}$ imply that $t>k^{3 / 4}-2$. Thus, (2) and (3) yield $t p_{2} \geq t p_{1}+2 t>n-2 k^{3 / 4}+4+2 k^{3 / 4}-$ $4=n$ and

$$
(t+1) p_{2}<(t+1) p_{1}+(t+1) c_{2} \ln k<n+k / 2+k^{3 / 4}+t c_{2} \ln k .
$$

But $t \leq n / p_{1}<2 n / k<2 c_{1} k / \ln k$. Put $\delta=1 / 2 k_{0}^{1 / 4}$. By (4) we have

$$
(t+1) p_{2}<n+k / 2+k^{3 / 4}+2 c_{1} c_{2} k<n+k \quad \text { if } \quad c_{1}<\left(\frac{1}{4}-\delta\right) / c_{2} .
$$

Now only the case $k^{1+c}<n+k<k^{7 / 4}$ remains to be considered. For such $n$ we prove that $\left(\begin{array}{c}n+k \\ k\end{array}\right)$ has a prime factor $p$ satisfying $k / 2<p<k / 2+k^{7 / 8}$. To see this, let $p$ be any prime in the interval $\left(k / 2, k / 2+k^{7 / 8}\right)$ and let $t_{p} p$ be the largest multiple of $p$ less than or equal to $n$. If $t_{p} p \leq n-2 k^{7 / 8}$, then $\left(t_{p}+2\right) p \leq n+k$, and hence $p \mid\left(\begin{array}{c}n+k \\ k\end{array}\right)$. Thus, a prime $p$ is unusable only if $n-2 k^{7 / 8}<t_{p} p \leq n$, that is, $\left(n-2 k^{7 / 8}\right) / t_{p}<p \leq n / t_{p}$. Thus, for fixed $t_{p}$ there are at most $2 k^{7 / 8} / t_{p}$ unusable primes with the same multiplier $t_{p}$. Next we estimate the number of possible values of $t_{p}$. Clearly, $t_{p}<n /(k / 2)$ and

$$
t_{p}>\frac{n-2 k^{7 / 8}}{k / 2+k^{7 / 8}}>\frac{2 n}{k+3 k^{7 / 8}} \text { provided that } n>2 k+6 k^{7 / 8} .
$$

Thus, the number of possible values of $t_{p}$ is at most

$$
\frac{2 n}{k}-\frac{2 n}{k+3 k^{7 / 8}}<\frac{6 n}{k^{9 / 8}},
$$

so the number of unusable primes is at most

$$
\frac{2 k^{7 / 8}}{t_{p}} \cdot \frac{6 n}{k^{9 / 8}}<6 k^{3 / 4}\left(1+\frac{3}{k^{1 / 8}}\right) .
$$

But in $\left(k / 2, k / 2+k^{7 / 8}\right)$ there are, by Ingham's result, more than $k^{7 / 8} /(c \ln k)$ primes, which is of higher order than $k^{3 / 4}$. Thus there are many usable primes.

This completes our proof that $g(k)>c_{1} k^{2} / \ln k$. In fact, our proof can be easily modified to show that if $n<c_{1} k^{2} / \ln k$, then there are more than 
$c_{3} k / \ln k$ primes in $(k / 2, k)$ which divide $\left(\begin{array}{c}n+k \\ k\end{array}\right)$. No doubt this still holds for much larger values of $N$, but we cannot prove this.

Theorem 2. If neither $k+1$ nor $k+2$ is prime, then $g(k) \geq(k+1) q-1$, where $q$ is the largest prime power divisor of $k+2$.

Proof. Suppose $\left(\begin{array}{l}N \\ k\end{array}\right)$ is good. Lemma 1 below implies that $N \equiv-1 \bmod (k+1)$ and $N \equiv-1$ or $-2 \bmod q$. Then $N \equiv-1$ or $k \bmod (k+1) q$. But $N>k$, so $N \geq(k+1) q-1$.

Corollary 1. If $k+1$ is composite and $k+2=p^{a}(a>1)$, then $g(k) \geq$ $k^{2}+3 k+1$.

Examples. $g(7) \geq 71, g(14) \geq 239(=!), g(23) \geq 599, g(25) \geq 701$.

Corollary 2. If $k+1$ is composite and $k+2=2 p^{a} \quad(a \geq 1)$, then $g(k) \geq$ $\left(k^{2}+3 k\right) / 2$.

Examples. $g(8) \geq 44(=!), g(20) \geq 230, g(24) \geq 324, g(32) \geq 560$, $g(44) \geq 1034$.

We confidently conjecture that $g(k)>k^{2}$ for $k>16$, with the notable exception $g(28)=284$. We conjecture that $g(k)>k^{3}$ for $k>35$, with a charming near exception $g(99)=5675499$. It would not surprise us if $g(k)>k^{5}$ for $k>100$.

Lemma 1. The prime $p$ does not divide $\left(\begin{array}{l}N \\ k\end{array}\right)$ if and only if each of the digits of $N$ base $p$ is greater than or equal to the corresponding digit of $k$ base $p$, or, equivalently, $N \bmod p^{a} \geq k \bmod p^{a}$ for all $a \leq b$ such that $p^{b-1} \leq k<p^{b}$, where $n \bmod m:=n-m\left\lfloor\frac{n}{m}\right\rfloor$.

The proof of Lemma 1 is easy and well known, but we include it for completeness.

Proof. If the digit in the $p^{a}$ column of $N$ base $p$ is greater than or equal to the corresponding digit of $k$ base $p$, then there are the same number of multiples of $p^{a}$ among $n+1, \ldots, n+k$ as there are among $1, \ldots, k$. If this holds for each $a$, then $p$ does not divide $\left(\begin{array}{l}N \\ k\end{array}\right)$. Otherwise, there are more multiples of $p^{a}$ among $n+1, \ldots, n+k$ than among $1, \ldots, k$, for some $a$, and $p$ divides $\left(\begin{array}{l}N \\ k\end{array}\right)$. For the alternative version, if $N \bmod p^{a} \geq k \bmod p^{a}$, then $n+1, \ldots, n+k$ have the same number of multiples of $p^{a}$ as $1, \ldots, k$ have, for each $a$, and thus $p$ does not divide the binomial coefficient $\left(\begin{array}{l}N \\ k\end{array}\right)$.

\section{LEAST PRIME FACTORS OF BINOMIAL COEFFICIENTS}

The main problem: Estimate $p\left(\left(\begin{array}{l}N \\ k\end{array}\right)\right)$, the least prime factor $p$ of $\left(\begin{array}{l}N \\ k\end{array}\right)$.

Case 1. $N>k^{2}$.

Conjecture. We conjecture [6] that in this case, $p\left(\left(\begin{array}{c}N \\ k\end{array}\right)\right) \leq N / k$ except for $\left(\begin{array}{c}62 \\ 6\end{array}\right)$.

If any $b_{i}$ is composite, then $p\left(\left(\begin{array}{l}N \\ k\end{array}\right)\right) \leq \sqrt{b_{i}} \leq \sqrt{N}<N / k$. If $\left(\begin{array}{l}N \\ k\end{array}\right)$ is not good, then $p\left(\left(\begin{array}{l}N \\ k\end{array}\right)\right) \leq k<N / k$, satisfying our conjecture. For the remainder of Case 1 , we will only consider good binomial coefficients.

To see why we must allow for $p\left(\left(\begin{array}{l}N \\ k\end{array}\right)\right)=N / k$, suppose

$$
a_{i}<k, \text { for all } i<k \text { and } a_{k}=k \text {. }
$$


Then $p\left(\left(\begin{array}{l}N \\ k\end{array}\right)\right)=N / k$ if and only if the $b_{i}$ are all prime. Sequences satisfying (5) were characterized in [2]. For a given $k$ the $a_{i}$ are always a permutation of $1, \ldots, k$ and the number of such permutations is always a power of 2 . For example, when $k=2459$, there are five operations which we call $2^{11}$ swap, $3^{7}$ swap, $7^{4}$ swap, 2459 swap (followed by symmetric flip), and 1229 +2 twin prime double swap, yielding 32 permutations which can be written immediately. A more mundane example is provided by $k=5$, which admits a $2^{2}$ swap, yielding just two permutations. When $n=210$ for instance, the $a_{i}$ are $1,4,3,2,5$ and the $b_{i}$ are $211,53,71,107,43$.

If none of the $b_{i}=1$ and $k \mid n+i$, then $p((n+i) / k) \leq(n+i) / k \leq N / k$. So our conjecture remains to be verified only for those good binomial coefficients where at least one of the $b_{i}=1$. If $N$ is very large compared to $k$ (for example, if $N>k$ !), then $b_{i}>1$ for all $i$.

Schinzel has conjectured that for every $k$ there is an $N \geq 2 k$ such that the $b_{i}$ are all prime. We note that $\left(\begin{array}{l}N \\ k\end{array}\right)$ can be the product of fewer than $k$ primes all greater than $k$. For example, when $k=25$ and $n=2080$, then $b_{10}=b_{13}=b_{20}=1$, and the other $b_{i}$ are all prime. Since $\left(\begin{array}{c}2105 \\ 25\end{array}\right)$ is good, it is the product of these 22 primes, each greater than 25 .

Definition 2. If $\left(\begin{array}{l}N \\ k\end{array}\right)$ is good, we call the number of $i$ such that $b_{i}=1$ the deficiency of $\left(\begin{array}{l}N \\ k\end{array}\right)$ and use the notation $d(N, k)$ or $d$ in context.

Thus $d(2105,25)=3$ and $d(215,5)=0$.

Theorem 3. If $\left(\begin{array}{l}N \\ k\end{array}\right)$ is good, and $N>c_{4} 2^{k} \sqrt{k}$ (where $c_{4}<0.4$ when $k \geq 94$ ), then $d(N, k)=0$. (For $k<94$, see Tables 2 and 3.)

The proof of Theorem 3 depends on the following lemma.

Lemma 2. If $\left(\begin{array}{l}N \\ k\end{array}\right)$ is good, then $a_{i} \mid i\left(\begin{array}{l}k \\ i\end{array}\right)$.

Proof of Lemma 2. We use the alternative form $a_{i}=a_{k-j}, 0 \leq j<k$. If $p^{a} \mid a_{k-j}$, then $p^{a} \mid n+k-j$, so $N \bmod p^{a}=j \bmod p^{a}$. Since $\left(\begin{array}{l}N \\ k\end{array}\right)$ is good, $j \bmod p^{a} \geq k \bmod p^{a}$ by Lemma 1 . Put $q p^{a} \leq j<(q+1) p^{a} ;$ there are $q$ multiples of $p^{a}$ among $1, \ldots, j$. But since $k \bmod p^{a} \leq j \bmod p^{a}$, there are $q+1$ multiples of $p^{a}$ among the $j+1$ numbers $k, k-1, \ldots, k-j$. The same argument shows that there is one more multiple of $p^{d}$ among $k, \ldots, k-j$ than among $1, \ldots, j$ for any $d \leq a$. Each $d \geq 1$ contributes a count of one to the power of $p$ dividing

$$
\frac{k(k-1) \cdots(k-j+1)(k-j)}{j !}=(k-j)\left(\begin{array}{l}
k \\
j
\end{array}\right),
$$

which is therefore a multiple of $p^{a}$. Thus, $a_{k-j} \mid(k-j)\left(\begin{array}{c}k \\ k-j\end{array}\right)$ for $0 \leq$ $j<k$.

Proof of Theorem 3. By Lemma 2, $a_{i} \leq i\left(\begin{array}{c}k \\ i\end{array}\right)$. The maximum for this bound occurs when $i=\lfloor(k+1) / 2\rfloor$. If $k$ is even, $a_{i} \leq(k / 2)\left(\begin{array}{c}k \\ k / 2\end{array}\right)<2^{k} \sqrt{k / 2 \pi}$, using Stirling's formula. For $k$ odd, $k \geq 95, a_{i}<0.42^{k} \sqrt{k}$. So if $N-k$ is larger than this maximum, then none of the $b_{i}$ equals 1 . 
TABLE 2. Binomial coefficients $\left(\begin{array}{l}N \\ k\end{array}\right)$ with deficiency $d>1$

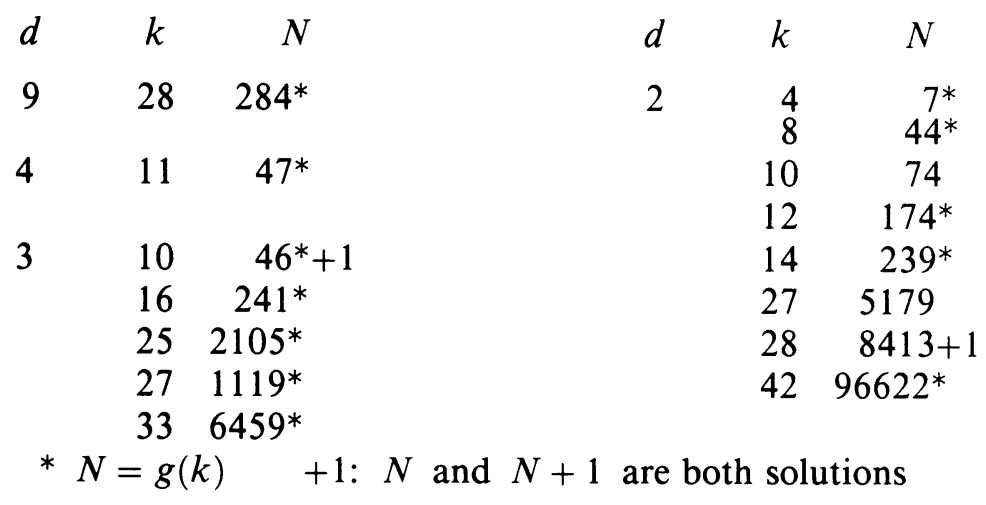

We ran a program (based on a more precise bound on $a_{i}$ ) to find binomial coefficients with positive deficiency for $k \leq 101$ and all possible $N$. Tables 2 and 3 give the results of our computer search.

Notice that in Table 3 below there is a positive deficiency at $k=100$ near the top of our search range. But for $k \geq 20$ our published remark [3, p. 523] that for each $k$ there seems to be an $N$ such that $d(N, k)=1$ is way off base. In fact, the first $k$ for which $d(N, k)=0$ for all good $\left(\begin{array}{l}N \\ k\end{array}\right)$ is 13 .

We conjecture that $k=42$ is the last $k$ with $d(N, k)>1$, and from looking at the tables, one gets the idea that there are only finitely many binomial coefficients with $d(N, k)>0$. The only values of $k \leq 42$ for which $d=0$ for all good $N$ are 13,20,21, 22, 24, 29, 31, 37. However, for $42<k \leq 101$ there are only 13 values of $k$ with $d(N, k)=1$ for some $N$, and none with $d>1$ for any $N$.

Notice also that whenever we found $d(N, k)>1$, then $d(g(k), k)>1$. There are 11 values of $k \leq 46$ with $d(g(k), k)=1$. We conjecture that $d(g(k), k)=0$ for $k>46$; this has been checked up to $k=149$.

If $N>k^{2}$, then $\left(\begin{array}{c}62 \\ 6\end{array}\right)$ is the only known exception to $p\left(\left(\begin{array}{l}N \\ k\end{array}\right)\right) \leq N / k$. If there were to be any further exceptional binomial coefficients $\left(\begin{array}{l}N \\ k\end{array}\right)$ in Case 1, the following four conditions would all have to be satisfied: $1 . d(N, k)>0$, so $k>101$. 2. $N \geq g(k)$. 3. If $b_{i} \neq 1$, then $b_{i}$ is prime. 4. If $b_{i} \neq 1$, then $a_{i} \leq k$.

Remark 1 . The probability that one of the $k$ consecutive integers of $\{n+i\}$ is divisible by a prime just larger than $k$ is close to 1 . Thus, if $N>k^{3}$, there will almost always be a prime factor less than or equal to $N / k$.

Case 2. $2 k \leq N \leq k^{2}$.

Lemma 3. We have $p(r) \mid\left(\begin{array}{c}r k \\ k\end{array}\right)$.

Proof. Let $p=p(r)$ and $p^{a} \| r k$. Then $r k \bmod p^{a}=0<k \bmod p^{a}$. Thus, by Lemma $1, p(r) \mid\left(\begin{array}{c}r k \\ k\end{array}\right)$. So at $N=k^{2},\left(\begin{array}{l}N \\ k\end{array}\right)$ has a prime factor $p(k) \leq k=$ $N / k$.

Remark 2. If $N=k^{2}-1$, then $\left.p\left(\begin{array}{l}N \\ k\end{array}\right)\right)<N / k$.

Proof. Let $p=p(k-1)$. Then $N \bmod p=0$ and $k \bmod p=1$. By Lemma 1, $p \mid\left(\begin{array}{l}N \\ k\end{array}\right)$, so $p\left(\left(\begin{array}{l}N \\ k\end{array}\right)\right) \leq k-1<N / k$. 
TABLE 3. Binomial coefficients with deficiency $d(N, k)=1$

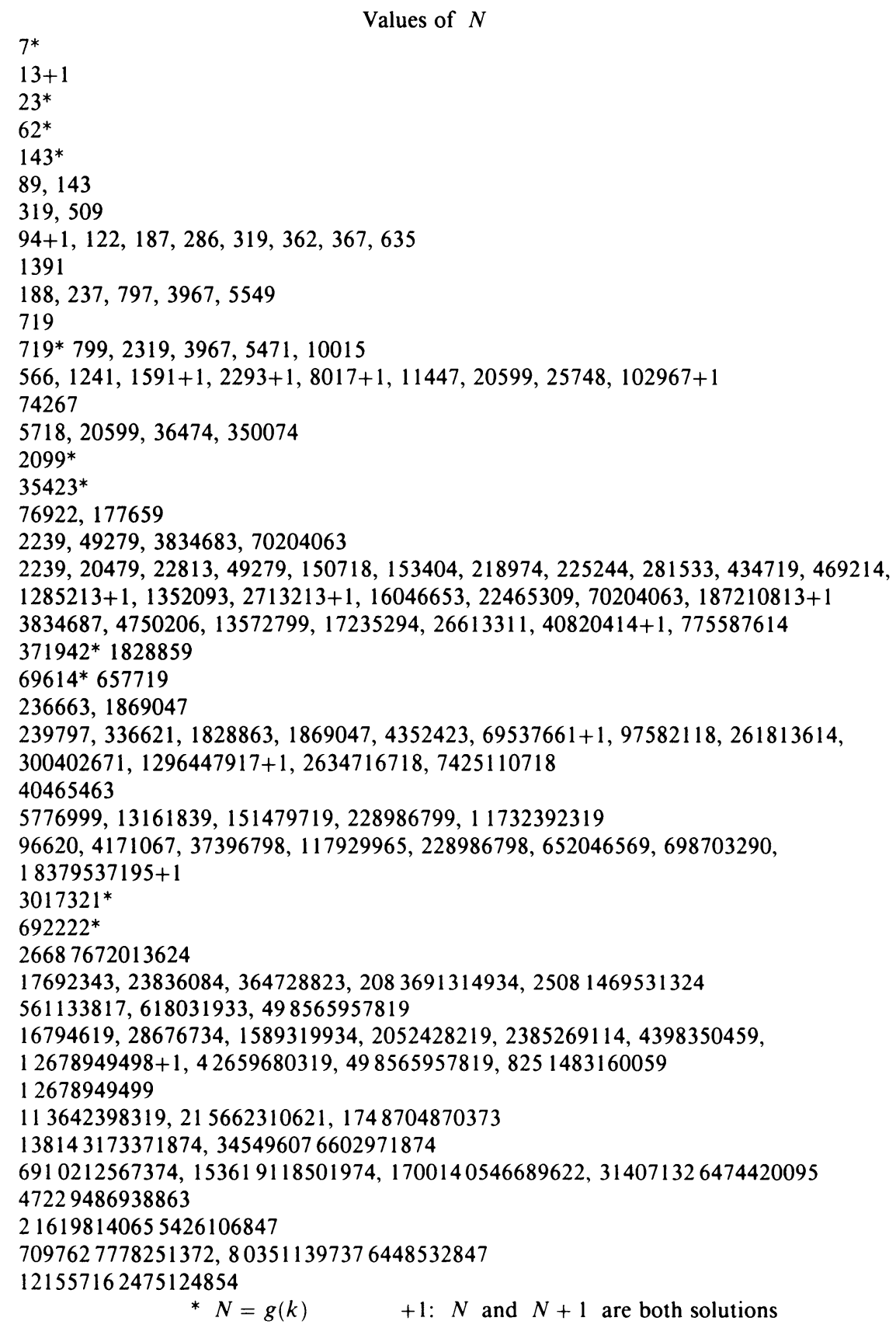


When $N / k$ is small, it is unacceptably small as a bound for $p\left(\left(\begin{array}{l}N \\ k\end{array}\right)\right)$. In fact, there are infinitely many binomial coefficients with $\left.p\left(\begin{array}{l}N \\ k\end{array}\right)\right)=3>N / k$ when $N<3 k$.

Theorem 4. For each $k>2$ there is an $N, 2 k \leq N<4 k$, such that $p\left(\left(\begin{array}{l}N \\ k\end{array}\right)\right)>$ $N / k$.

Proof. Let $2^{r-1} \leq k<2^{r}$ and let $N=2^{r}+k$. Then by Lemma $1,\left(\begin{array}{l}N \\ k\end{array}\right)$ is odd, since $N \equiv k \bmod 2^{a}$ for $a=1, \ldots, r$. But if $\left(\begin{array}{c}N \\ k\end{array}\right)$ is odd and $2 k<N<3 k$, then $p\left(\left(\begin{array}{l}N \\ k\end{array}\right)\right) \geq 3>N / k$. Now there will always be an $N=2^{r}+k, 2 k<$ $2^{r}+k<3 k$, except when $2^{r}=2 k$. But when $2^{r-1}=k$, then $\left(\begin{array}{l}N \\ k\end{array}\right)$ is odd for all values of $N$ such that $3 k \leq N<4 k$. Now take $N=3^{s}+k, 2 \cdot 3^{s}-1$ or $2 \cdot 3^{s}+k$, whichever is in the region. By Lemma 1, 3 does not divide $\left(\begin{array}{l}N \\ k\end{array}\right)$. So $p\left(\left(\begin{array}{l}N \\ k\end{array}\right)\right) \geq 5>N / k$.

We now combine Cases 1 and 2 .

Definition 3. If $p\left(\left(\begin{array}{l}N \\ k\end{array}\right)\right)>N / k$, then $\left(\begin{array}{l}N \\ k\end{array}\right)$ is said to be exceptional.

It would be very interesting if someone could prove our conjecture that the number of exceptional $\left(\begin{array}{l}N \\ k\end{array}\right)$ with $p>17$ is finite. We wrote a program to find all exceptional $\left(\begin{array}{l}N \\ k\end{array}\right)$ where $p>5$ and $k \leq 12000$. The most unusual exception is for $p=29: p\left(\left(\begin{array}{c}284 \\ 28\end{array}\right)\right)=29$. We have found one exception for $p=23$ : $p\left(\left(\begin{array}{c}474 \\ 66\end{array}\right)\right)=23$. There appear to be exactly two exceptions for $p=19$ : $\left(\begin{array}{c}62 \\ 6\end{array}\right)$ and $\left(\begin{array}{c}959 \\ 56\end{array}\right)$.

We have two further conjectures:

1. The only exceptional $\left(\begin{array}{l}N \\ k\end{array}\right)$ with $p>17$ are these four.

2. $p\left(\left(\begin{array}{l}N \\ k\end{array}\right)\right) \leq N / k$ if $N>17 \frac{1}{8} k$.

Notice that 1 . is a bit stronger and implies 2 .

We have found eight exceptional $\left(\begin{array}{l}N \\ k\end{array}\right)$ with $p=17:\left(\begin{array}{c}241 \\ 16\end{array}\right),\left(\begin{array}{c}439 \\ 33\end{array}\right),\left(\begin{array}{c}317 \\ 56\end{array}\right)$, $\left(\begin{array}{l}482 \\ 130\end{array}\right),\left(\begin{array}{l}998 \\ 256\end{array}\right),\left(\begin{array}{c}998 \\ 260\end{array}\right),\left(\begin{array}{c}14273 \\ 896\end{array}\right),\left(\begin{array}{c}13277 \\ 900\end{array}\right)$, and two near misses: $\left(\begin{array}{c}239 \\ 14\end{array}\right)$ and $\left(\begin{array}{c}956 \\ 56\end{array}\right)$.

Our program gave only one output for $331<k<625$, namely, $p\left(\left(\begin{array}{c}3574 \\ 406\end{array}\right)\right)=$ 13 , and no $p>13$ for any $k$ other than those already listed. Thus, at this point in time, it is still possible that $p\left(\left(\begin{array}{l}N \\ k\end{array}\right)\right) \leq \max (N / k, 13)$, with the twelve exceptions listed above.

\section{A RElAted PROBlem}

We now turn our attention to a related problem. We study the number of indices $i$ where $b_{i}=1$ without requiring that $\left(\begin{array}{l}N \\ k\end{array}\right)$ be good.

Theorem 5. Assume $N \geq 2 k$ and denote by $f(N, k)$ the number of indices $i$ for which $b_{i}>1$. Then $f(N, k) \geq(1-\epsilon) \pi(k)$ for $k>k_{0}(\epsilon)$.

Proof. We use the strong form of the prime number theorem: the number of primes $p$ such that $N-k<p \leq N$ is greater than $(1-\epsilon) \pi(k)$ if $N<k^{1+c}$ for any $c \leq 1 / 3$. This immediately gives the theorem for $2 k \leq N<k^{4 / 3}$.

In fact, the contribution to $b_{i}>1$ from primes $k<p \leq N / 2$ becomes significant even when $N=(2+\delta) k$. When $N=3 k$, use the quoted theorem for $k<p \leq N / 2$ and $2 k<p \leq N$ to get $f(N, k) \geq\left(\frac{3}{2}-\epsilon\right) \pi(k)$ for large $k$, and similarly one can get $f(N, k)>(1+\mu) \pi(k)$ when $N \geq(2+\delta) k$. 
Now if $N \geq k^{4 / 3}$, we will show that $f(N, k)>k / 8$ for $k$ large enough. First observe that $\left(\begin{array}{l}N \\ k\end{array}\right) \geq N^{k} / k^{k}$. The well-known result that $p^{\alpha} \leq N$ when $p^{\alpha} \|\left(\begin{array}{l}N \\ k\end{array}\right)$ follows easily from Lemma 1 . Thus we have

$$
N^{k} / k^{k} \leq\left(\begin{array}{l}
N \\
k
\end{array}\right)<N^{\pi(k)} N^{f(N, k)} .
$$

Now $N^{3 / 4} \geq k$ and, for $k>10^{4}, \pi(k)<k / 8$. Thus, from (6), using $N / k \geq$ $N^{1 / 4}$, we get $N^{k / 4} \leq N^{k} / k^{k}<N^{k / 8} N^{f(N, k)}$ and thus $f(N, k)>k / 8>\pi(k)$, which finishes the proof of the theorem.

It would be quite difficult to give good explicit inequalities for $f(N, k)$.

Corollary 3. If $N \geq 2 k$, there are at least $(1-\epsilon) \pi(k)$ primes greater than $k$ dividing $\left(\begin{array}{l}N \\ k\end{array}\right)$. In fact, when $N \leq k^{2}$, the count of primes greater than $k$ is $f(N, k)$, since all $b_{i}$ are prime.

Notice that $f(21,10)=3<\pi(20)-\pi(10)=4$. Is it true that for every $t$ there are integers $N$ and $k$ for which $f(N, k) \leq \pi(2 k)-\pi(k)-t$ ? For $f(213,100)$ we can take $t=3$.

We conjecture that there are examples with arbitrarily large $t$. Suppose there is a large gap in the primes between $p_{r}$ and $p_{r+1}$. Take $N=p_{r+1}-1$ and $2 k=p_{r}+1$. Then $f(N, k)$ is the number of primes between $N-k$ and $N$, plus the number of primes between $k$ and $N / 2$. Since $\pi(N)=\pi(2 k)$, we get

$$
f(N, k)=\pi(2 k)-\pi(k)-(\pi(N-k)-\pi(N / 2)) .
$$

As an example, the first gap of 320 between consecutive primes is listed by Lander and Parkin [4]. Put $N=2300942868$ and $k=1150471275$. The primes between $N / 2$ and $N-k$ are $11.50471000+297,307,319,369,373$, 393, 417, giving an example with $t=7$.

From the strong form of the prime number theorem, we deduce that only the interval $2 k<N<2 k+k^{c}$ has to be searched. No doubt the only possible examples with large $t$ lie in the interval $2 k<N<2 k+c \ln k$, where $c=$ $c(t)>0$.

\section{ACKNOWLEDGMENT}

We thank the referee for his careful reading.

\section{BIBLIOGRAPHY}

1. E. F. Ecklund, P. Erdös, and J. L. Selfridge, A new function associated with the prime factors of $\left(\begin{array}{l}n \\ k\end{array}\right)$, Math. Comp. 28 (1974), 647-649.

2. P. Erdös, C. B. Lacampagne, and J. L. Selfridge, Prime factors of binomial coefficients and related problems, Acta Arith. 49 (1988), 507-523.

3. A. E. Ingham, On the differences between consecutive primes, Quart. J. Math. Oxford 8 (1937), 255-266.

4. L. J. Lander and T. R. Parkin, On first appearance of prime differences, Math. Comp. 21 (1967), 483-488.

5. R. Scheidler and H. C. Williams, A method of tabulating the number-theoretic function $g(k)$, Math. Comp. 59 (1992), 251-257. 
6. J. L. Selfridge, Some problems on the prime factors of consecutive integers, Abstract 747-10-9, Notices Amer. Math. Soc. 24 (1977), A456-A457.

(P. Erdős) c/o Dr. R. L. Graham, AT\&T Bell Laboratories, Murray Hill, New Jersey 07974

Office of Research, U. S. Department of Education, Washington, DC 20008

E-mail address: clacampa@nsf.gov

Department of Mathematics, Northern Illinois University, DeKalb, Illinois 60115 